\title{
Measure Solutions of the Steady Boltzmann Equation in a Slab
}

\author{
Leif Arkeryd ${ }^{1}$, Carlo Cercignani ${ }^{2}$ and Reinhard Illner ${ }^{3}$ \\ Department of Mathematics, Chalmers Institute of Technology, Goeteborg, Sweden \\ 2 Dipartimento di Matematica, Politecnico di Milano, I-20133 Milano, Italy \\ ${ }^{3}$ Department of Mathematics, University of Victoria, P.O. Box 3045, Victoria, B.C. V8W 3P4, \\ Canada
}

Received October 25, 1990; in revised form March 29, 1991

\begin{abstract}
It is shown that the steady Boltzmann equation in a slab $[0, a]$ has solutions $x \rightarrow \mu_{x}$ such that the ingoing boundary measures $\mu_{0 \mid\{\xi>0\}}$ and $\mu_{a \mid\{\xi<0\}}$ can be prescribed a priori. The collision kernel is truncated such that particles with small $x$-component of the velocity have a reduced collision rate.
\end{abstract}

\section{Introduction}

Throughout this paper, $v=(\xi, \eta, \zeta) \in \mathbb{R}^{3}$ will denote a velocity vector with $x-, y$ - and $z$-components $\xi, \eta$ and $\zeta$ respectively. $x$ is the (one-dimensional) position in the interval $[0, a]$. This interval is also referred to as a "slab."

For two velocities $v, w \in \mathbb{R}^{3}$ and a collision parameter $n \in S^{2}$, we define the collision transformation

by

$$
J:(v, n, w) \rightarrow\left(v^{\prime},-n, w^{\prime}\right)
$$

$$
\begin{aligned}
v^{\prime} & =v-n(n, v-w), \\
w^{\prime} & =w+n(n, v-w) .
\end{aligned}
$$

Here, $(n, v-w)$ denotes the Euclidean inner product in $\mathbb{R}^{3} . J$ is an involution $\left(J^{2}=\right.$ id) and preserves momentum and energy. It is also well-known (and easily checked) that $\left\|v^{\prime}-w^{\prime}\right\|=\|v-w\|$ and $|(n, v-w)|=\left|\left(n, v^{\prime}-w^{\prime}\right)\right|$, so the collision kernel $B(n, v-w)$, which in effect only depends on $\|v-w\|$ and $|(n, v-w)|$, is invariant under the action of $J$.

We are concerned with the steady Boltzmann equation in the slab $0 \leqq x \leqq a$, for $f=f(x, v)$,

$$
\xi \cdot \frac{d}{d x} f=C(f, f)
$$


with boundary conditions

$$
\begin{array}{lll}
f(0, v)=f_{0}(v) & \text { if } \quad \xi>0, \\
f(a, v)=f_{a}(v) & \text { if } \quad \xi<0 .
\end{array}
$$

The collision operator $C(f, f)$ is

$$
C(f, f)(x, v)=\int_{\mathbb{R}^{3}} \int_{S^{2}} B(n, v-w)\left[f^{\prime} f_{*}^{\prime}-f f_{*}\right] d n d w
$$

with $f^{\prime}=f\left(x, v^{\prime}\right), f_{*}^{\prime}=f\left(x, w^{\prime}\right)$ and $f_{*}=f(x, w)$.

For the time being we impose no restrictions on the collision kernel; we need some truncations later on.

Problem (1.2-3) models a kinetic layer between two walls at $x=0$ and $x=a$, where the ingoing densities are prescribed. Our objective in this paper is to decide whether this problem has, in some sense, a nonnegative solution. To this end, we find it necessary to extend the functions admissible as solutions to measure valued functions of $x$,

$$
\begin{aligned}
x & \rightarrow \mu_{x}, \\
{[0, a] } & \rightarrow M .
\end{aligned}
$$

Here, $M$ denotes the set of bounded measures on $\mathbb{R}^{3}$.

If $x \rightarrow f(x, v) \in L^{1}\left(\mathbb{R}^{3}\right)$ were a classical solution of (1.2), we could write $d \mu_{x}(v)=f(x, v) d v$, but we will see that (1.2-3) admits a reasonable measure solution concept which does not require that $\mu_{x}$ be absolutely continuous for any $x$. In particular, this concept permits general finite measures as boundary values.

For mass- and momentum-conserving discrete velocity models for which $\xi_{i} \neq 0$ for all the admissible velocities $v_{i}=\left(\xi_{i}, \eta_{i}, \zeta_{i}\right)$, the counterpart to problem (1.2-3), and also (1.2) with many other boundary conditions, were shown in [1] to always have a solution. Here we solve the problem for the full Boltzmann equation, but we truncate the collision kernel in the collision operator such that the particles with small $|\xi|$ do not at all interact or have at least a reduced rate of interaction. This is unphysical, but necessary in our method to prove a priori estimates. The case where particles are allowed to interact freely even if one of them has a velocity $v=(\xi, \eta, \zeta)$ with arbitrarily small $|\xi|$ is open.

The plan of the paper is as follows. In Sect. 2, we introduce a measure theoretic formulation of problem $(1.2-3)$. The rationale behind this is that a measure formulation makes the problem accessible to the simple compactness criteria which are available in the cone of bounded measures (by the Alaoglu theorem, a set of bounded measures is weak-* relatively compact if it is uniformly bounded - see [6]). In Sect. 3, we introduce two truncations to the collision kernel: A rather crude truncation, which will make the resulting problem accessible to Tychonoff's fixed point theorem, and a second, less restrictive truncation, which is our ultimate target.

In Sect. 4 we review Tychonoff's fixed point theorem (see [6, 8-10]) and apply it to show that the boundary value problem with the crude truncation has a measure solution. The discussion includes the introduction of the underlying function space, a corollary to Tychonoff's theorem due to Schaefer [8] and the a priori estimates needed to apply this theorem. Finally, in Sect. 5, we use an 
approximation procedure to show that the problem with the less restrictive truncation also has a measure solution.

A few remarks are in order. First, we note that the analysis here does not require an entropy functional. In fact, for the steady problem $(1.2-3)$, the $H$-Theorem takes the form

$$
\frac{d}{d x} \int \xi \cdot f \cdot \ln f d v \leqq 0,
$$

and because the multiplier $\xi$ here does not have a definite sign, we cannot use entropy to get even weak compactness in $L^{1}$. It was this lack of weak compactness in $L^{1}$ which inspired us to look for a measure formulation of $(1.2-3)$.

The results presented here are unsatisfactory for two reasons: The truncations which we introduce are unphysical, and the solutions obtained may not be unique. While there is at least some hope to eventually remove the truncations imposed here entirely, the uniqueness question is much more serious. Not only does Tychonoff's theorem not imply uniqueness, the solution for our boundary value problem may just not be unique.

We mention some related papers on kinetic boundary layers. In [3], the halfspace problem $(a=\infty)$ was treated for discrete velocity models. A two dimensional boundary value problem was solved for the standard 4-velocity model in [2], and tentative steps to solve general two-dimensional boundary value problems were taken in [5]. A useful classification of the well-posedness of boundary value problems for the linearized Boltzmann equation was given by Coron, Golse and Sulem in [4].

\section{Measure Formulation}

We return to (1.2-3). To pass to a measure formulation, choose a test function $\varphi(x, v)$, bounded and continuous, such that $\partial_{x} \varphi(x, v) / \xi$ is continuous and such that $\varphi$ is Lipschitz continuous with respect to $v$ (with a Lipschitz constant not depending on $x$ ) and has compact support. In addition, we require that

$$
\begin{array}{ll}
\varphi(0, v)=0 \quad \text { if } \quad \xi<0 \\
\varphi(a, v)=0 \quad \text { if } \quad \xi>0
\end{array}
$$

We call such test functions "admissible."

Multiply (1.2) by $\varphi$, integrate $\int_{0}^{a} \int_{\mathbb{R}^{3}} d v d x$, integrate by parts with respect to $x$, apply the collision transformation and use the boundary condition (1.3). The result is

$$
\begin{aligned}
& -\int_{0}^{a} \int_{\xi} \xi \cdot f(x, v) \frac{\partial}{\partial x} \varphi(x, v) d v d x-\int_{\xi>0} \xi \cdot f_{0}(v) \varphi(0, v) d v+\int_{\xi<0} \xi \cdot f_{a}(v) \varphi(a, v) d v \\
& =\int_{0}^{a} \int_{v} \int_{w} \int_{S^{2}}\left(\varphi\left(x, v^{\prime}\right)-\varphi(x, v)\right) B(n, v-w) f(x, v) f(x, w) d n d w d v d x
\end{aligned}
$$


or, in measure notation,

$$
\begin{aligned}
& -\int_{0}^{a} \int_{0} \xi \cdot \frac{\partial}{\partial x} \varphi(x, v) d \mu_{x}(v) d x-\int_{\xi>0} \varphi(0, v) \cdot \xi d \mu_{0}^{+}(v)+\int_{\xi<0} \varphi(a, v) \cdot \xi d \mu_{a}^{-}(v) \\
& =\int_{0}^{a} \iint_{v} \int_{S^{2}}\left(\varphi^{\prime}-\varphi\right) B(n, v-w) d n d \mu_{x}(v) d \mu_{x}(w) d x .
\end{aligned}
$$

$\mu_{0}^{+}$and $\mu_{a}^{-}$are, of course, the data at $x=0$ and $x=a$, interpreted as measures on $\xi>0$ and $\xi<0$ respectively. We can drop the condition that $\mu_{0}^{+}$and $\mu_{a}^{-}$be absolutely continuous - it is not necessary for the sequel.

Let the measure $d M_{x}(v, n, w)$ on $\mathbb{R}^{3} \times S^{2} \times \mathbb{R}^{3}$ be defined by

$$
d M_{x}(v, n, w)=d n d \mu_{x}(v) d \mu_{x}(w) .
$$

Because the collision transformation $J$ is involutive $\left(J^{-1}=J\right)$, the right-hand side of (2.2) can be rewritten as

$$
\int_{0}^{a} \int_{v} \int_{w} \int_{S^{2}} \varphi(x, v) B(n, v-w)\left[d\left(M_{x} \circ J\right)-d M_{x}\right](v, n, w) d x .
$$

Definition 2.1. Let $M=M\left(\mathbb{R}_{v}^{3}\right)$ be the cone of bounded measures on $\mathbb{R}_{v}^{3}$, endowed with the weak-* topology (i.e. $\mu_{n} \underset{w^{*}}{\longrightarrow} \mu$ if $\int \varphi \mu_{n} \rightarrow \int \varphi d \mu$ for each continuous $\varphi$ with compact support). A measure-valued function

$$
\begin{aligned}
{[0, a] } & \rightarrow M\left(\mathbb{R}_{v}^{3}\right), \\
x & \rightarrow \mu_{x}
\end{aligned}
$$

is called a measure solution of (1.2-3) if $x \rightarrow \mu_{x}$ is continuous (with respect to the weak-* topology on $\left.M\left(\mathbb{R}_{v}^{3}\right)\right)$ and (2.2) holds for all admissible test functions. We have automatically that $\mu_{0 \mid\{v ; \xi>0\}}=\mu_{0}^{+}$and $\mu_{a \mid\{v ; \xi<0\}}=\mu_{a}^{-}$.

We will assume throughout that $\mu_{0}^{+}$and $\mu_{a}^{-}$are such that all the integrals

$$
\int_{\xi>0} \xi d \mu_{0}^{+}(v), \int_{\xi<0}|\xi| \mu_{a}^{-}(v), \int_{\xi>0} \xi \cdot v^{2} d \mu_{0}^{+}(v) \text { and } \int_{\xi<0}|\xi| v^{2} d \mu_{a}^{-}(v)
$$

are finite.

Remark. We will have to address the problem that the set defined by $\xi=0$, while a set of Lebesgue measure zero, may not be a null set for the measures $\mu_{x}$. This problem will be solved by the truncations, which assure that the truncated collision kernel make no contributions to this set.

\section{Assumptions on the Collision Kernel and Truncations}

For $v=(\xi, \eta, \zeta) \in \mathbb{R}^{3}$ and $n \in S^{2}$, let $n$ be represented by the polar angle $\Phi$ (with polar axis along $v-w$ ) and the azimuthal angle $\theta$. We assume that

$$
B(n, v-w)=|v-w|^{\beta} h(\Phi),
$$

with $h$ integrable on $[0, \Pi]$ and $-1 \leqq \beta \leqq 0$, i.e. soft forces inversely proportional to the $j^{\text {th }}$ power of the distance, $3 \leqq j<5$. The condition $\beta \geqq-1$ is dictated by 
the presence of $\varphi^{\prime}-\varphi$ in the collision term: We must have

$$
|\varphi(x, v-n(n(v-w)))-\varphi(x, v)| \cdot|v-w|^{\beta}
$$

locally bounded as a function of $v$ and $w$; this is true, due to the Lipschitz continuity of $\varphi$, if $\beta \geqq-1$.

3.1 A Minimal Truncation. We introduce a factor $\chi_{\varepsilon}\left(v, w, v^{\prime}, w^{\prime}\right)$ into the collision term which serves the necessity to eliminate collisions between particles whose velocities have small $x$-component. Specifically, let $\varepsilon>0$ be arbitrary but fixed and let

$$
\chi_{\varepsilon}\left(v, w, v^{\prime}, w^{\prime}\right)=\left(\begin{array}{l}
1 \text { if } \min \left\{|\xi|,\left|\xi_{*}\right|,\left|\xi^{\prime}\right|,\left|\xi_{*}^{\prime}\right|\right\} \geqq \varepsilon \\
\text { such that } \chi_{\varepsilon} /\left(\xi^{2} \xi_{*}^{2}\right) \text { and } \chi_{\varepsilon} /\left(\xi^{\prime 2} \xi_{*}^{\prime 2}\right) \\
\text { are bounded otherwise }\left(\text { e.g., } \chi_{\varepsilon}=0\right) .
\end{array}\right.
$$

Here, $\xi_{*}, \xi^{\prime}$ and $\xi_{*}^{\prime}$ denote the $x$-components of $w, v^{\prime}$ and $w^{\prime}$ respectively. We also assume that $\chi_{\varepsilon}$ is invariant under the collision transformation. Let

$$
B_{\varepsilon}(v, n, w)=B(n, v-w) \cdot \chi_{\varepsilon}\left(v, w, v^{\prime}, w^{\prime}\right),
$$

and let $C_{\varepsilon}$ be the collision operator with $B$ replaced by $B_{\varepsilon}$. Our ultimate target in this paper is to show that the problem

$$
\begin{aligned}
\xi \frac{d}{d x} \mu_{x} & =C_{\varepsilon}\left(\mu_{x}, \mu_{x}\right), \\
\left.\mu_{0}\right|_{\{\xi>0\}} & =\mu_{0}^{+},\left.\quad \mu_{a}\right|_{\{\xi<0\}}=\mu_{a}^{-}
\end{aligned}
$$

admits a measure solution. We need a cruder truncation in preparation for this result.

3.2 A Crude Truncation. The truncation parameter $\varepsilon>0$ will be kept fixed once and for all. Now choose $\delta>0$, and let

$$
k_{\delta}=\left(\begin{array}{ll}
1, & \text { if } v^{2}+w^{2} \leqq \delta^{-2}, \quad \min \left\{|\xi|,\left|\xi_{*}\right|,\left|\xi^{\prime}\right|,\left|\xi_{*}^{\prime}\right|\right\}>\delta \text { and }|v-w|>\delta \\
0 & \text { otherwise (if one of the three above conditions is violated). }
\end{array}\right.
$$

Let $B^{\delta}=B_{\varepsilon} \cdot k_{\delta}$, and $C^{\delta}$ be the collision operator with $B$ replaced by $B^{\delta}$. The problem which we first solve is

$$
\begin{aligned}
\xi \frac{d}{d x} \mu_{x} & =C^{\delta}\left(\mu_{x}, \mu_{x}\right), \\
\left.\mu_{0}\right|_{\{\xi>0\}} & =\mu_{0}^{+},\left.\quad \mu_{a}\right|_{\{\xi<0\}}=\mu_{a}^{-} .
\end{aligned}
$$

\section{Tychonoff's Theorem and Measure Solutions of the Crudely Truncated Problem}

We begin this section by reviewing Tychonoff's theorem $([6,10])$ and a useful consequence due to Schaefer [8]. 
Theorem 4.1 (Tychonoff). Let $A$ be a non-empty compact convex subset of a locally convex, complete Hausdorff vector space $X$. If $T: A \rightarrow A$ is continuous, then $T$ has a fixed point.

Lemma 4.2 (Schaefer). Let $X$ be a locally convex complete Hausdorff vector space. If $K \subset X$ is convex and closed and $\varphi: K \rightarrow K$ is continuous and such that $\varphi(K)$ is relatively compact, then $\varphi$ has a fixed point in $K$.

Proof. Let $H=\varphi(K) \subset K$. The closed convex hull $H^{*}$ of $H$ is compact, and $H^{*} \subset K, \varphi\left(H^{*}\right) \subset H \subset H^{*}$. Therefore, $H^{*}$ satisfies the conditions of Tychonoff's theorem. q.e.d.

We prepare to apply these results to the problem (3.3-4). Let $X=C([0, a] ; M)$ be the cone of all continuous functions $[0, a] \rightarrow M$. Because the difference between two measures is in general not a measure anymore (but rather a signed measure), $X$ is not a linear space. However, Tychonoff's theorem and Lemma 4.2 can both be applied to $X$.

For $\mu \in M$, we write $\langle\mu, \varphi\rangle \underset{\text { def. }}{=} \int \varphi(v) d \mu(v)$. Elements of $X$ will be written as $x \rightarrow \mu_{x}$, or simply $\mu$.

Definition. $C_{\infty}\left(\mathbb{R}^{3}\right)$ will denote the Banach space of all continuous real-valued functions which vanish at $\infty$, endowed with the topology of uniform convergence. $A$ subset $E$ of $X$ is said to be (uniformly) equicontinuous if the set of real-valued functions $\left\{x \rightarrow\left\langle\mu_{x}, \varphi\right\rangle ; \mu_{.} \in E\right\}$ is (uniformly) equicontinuous for each $\varphi \in C_{\infty}\left(\mathbb{R}^{3}\right)$.

It is well-known [6] that every continuous functional on $C_{\infty}$ comes from a finite measure $\mu \in M$, and the weak-* topology on $M$ is the weakest topology such that $\mu \rightarrow\langle\mu, \varphi\rangle$ is continuous for any $\varphi \in C_{\infty}$. By the Banach-Alaoglu theorem, any bounded subset of $M$ is relatively compact; as $C_{\infty}$ is separable, the weak-* topology on bounded subsets of $M$ is metrizable, and so relative compactness and sequential relative compactness are equivalent (see, e.g. Royden [7]). This implies the Ascoli-Arzela Theorem for the current setting:

Lemma 4.3 (Ascoli-Arzela). A uniformly bounded and uniformly equicontinuous subset $E \subset X$ contains a convergent subsequence $\mu_{.}^{n} \rightarrow \mu_{.}$.

We finally note that $X$ is a locally convex and complete Hausdorff space (a separating family of seminorms is given by

$$
\rho_{\varphi}\left(\mu_{.}\right)=\sup _{x}\left|\left\langle\mu_{x}, \varphi\right\rangle\right|, \quad \varphi \in C_{\infty} ;
$$

completness is readily verified). Sometimes, we will use the notation $\left\|\mu_{.}\right\| \underset{\text { def. }}{=} \sup _{x \in[0, a]} \int d \mu_{x}(v)$.

We return to the setting of Sect. 3.2. The collision operator $C^{\delta}$ can be written, in a natural way, as

$$
C^{\delta}(f, f)=Q^{\delta}(f, f)-f R^{\delta}(f)
$$

or, in measure notation, as

$$
C^{\delta}\left(\mu_{x}, \mu_{x}\right)=Q^{\delta}\left(\mu_{x}, \mu_{x}\right)-R^{\delta}\left(\mu_{x}\right) \mu_{x}
$$


The function $R^{\delta}\left(\mu_{x}\right)(v)$ is

$$
R^{\delta}\left(\mu_{x}\right)(v)=\int_{\mathbb{R}^{3}} \int_{S^{2}} B^{\delta}(v, n, w) d n d \mu_{x}(w),
$$

and $Q^{\delta}\left(\mu_{x}, \mu_{x}\right), R^{\delta}\left(\mu_{x}\right) \mu_{x}$ are measures defined by

$$
\left\langle Q^{\delta}\left(\mu_{x}, \mu_{x}\right), \varphi\right\rangle=\iint_{v} \int_{n} B^{\delta}(v, n, w) \varphi(v) d\left(M_{x} \circ J\right),
$$

with $d M_{x}=d n d \mu_{x} \times d \mu_{x}$, and

$$
\left\langle R^{\delta}\left(\mu_{x}\right) \mu_{x}, \varphi\right\rangle=\left\langle\mu_{x}, R^{\delta}\left(\mu_{x}\right) \varphi\right\rangle .
$$

$B^{\delta}$ is uniformly bounded by construction $\left(B^{\delta} \leqq C(\delta)\right)$, so

$$
\left\|R^{\delta}\left(\mu_{x}\right)(v)\right\| \leqq 4 \pi C(\delta) \int d \mu_{x}(w) .
$$

Choose $R>0$ such that $\int_{\xi>0} d \mu_{0}^{+} \leqq R, \int_{\xi<0} d \mu_{a}^{-} \leqq R$, and let $B_{R}(0) \subset X$ be the set of

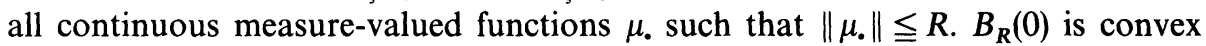
and closed.

We will find a solution of the crudely truncated problem (3.3-4) as a fixed point of a suitably constructed operator on $B_{R}(0)$, where $R$ is to be large enough.

Analogous to the method in [1], let, for any $\mu_{0} \in X, \rho(x)=\rho\left[\mu_{0}\right](x)=\int d \mu_{x}(v)$, $j(x)=j\left[\mu_{.}\right](x)=\int \xi d \mu_{x}(v)$ and $p(x)=p\left[\mu_{.}\right](x)=\int \xi^{2} d \mu_{x}(v)$.

For $\tau \geqq 4 \pi C(\delta)$, we define operators

if:

$$
T(\tau): B_{R}(0) \rightarrow X \quad \text { by } \quad v_{\bullet}=T(\tau) \mu_{.}
$$

$$
\begin{gathered}
\left.v_{0}\right|_{\{\xi>0\}}=\mu_{0}^{+},\left.\quad v_{a}\right|_{\{\xi<0\}}=\mu_{a}^{-} \\
\xi \cdot \frac{d}{d x} v_{x}=0 \text { for }|\xi| \leqq \delta, \quad|\xi| \geqq 1 / \delta
\end{gathered}
$$

and

$$
\xi \cdot \frac{d}{d x} v_{x}+\tau \rho\left[\mu_{.}\right](x) v_{x}=Q^{\delta}\left(\mu_{x}, \mu_{x}\right)+\tau \rho\left[\mu_{.}\right](x) \mu_{x}-R^{\delta}\left(\mu_{x}\right) \mu_{x}
$$

for $\delta<|\xi|<1 / \delta$.

We need to discuss whether $v_{\text {. is }}$ well defined. Clearly, (4.4) and (4.5) define $\left.v_{x}\right|_{\{|\xi| \leqq \delta\}}$ and $\left.v_{x}\right|_{\{|\xi| \geqq 1 / \delta\}}$ (we simply set $v_{x}\{\xi=0\}=0$ ) by, e.g. $\left.v_{x}\right|_{\{0<\xi \leqq \delta\}}=\left.\mu_{0}^{+}\right|_{\{0<\xi \leqq \delta\}}$. The right-hand side of (4.6) defines, for any $x \in[0, a]$, a measure because $R^{\delta}\left(\mu_{x}\right) \leqq$ $\tau \rho\left[\mu_{\text {. }}\right](x)$ for $\tau \geqq 4 \pi C(\delta)$. By our truncation from Sect. 3.2, the measures $Q^{\delta}\left(\mu_{x}, \mu_{x}\right)$ and $R^{\delta}\left(\mu_{x}\right) \mu_{x}$ are compactly supported in $\delta<|\xi|<1 / \delta$, so it is enough to show that (4.6) defines a measure function $v$. which is well defined and continuous on functions $\varphi \in C_{\infty}$ which are supported in this set.

Lemma 4.4. The boundary value problem (4.4), (4.6) has a unique solution $v_{.}$, and the mapping $\mu_{0} \rightarrow v_{0}$ is continuous from $B_{R}(0)$ into $X$.

Proof. $v$. can be constructed explicitly by following the solution method for linear differential equations. We leave the easy details to the reader.

It is evident that $v_{0}=T(\tau) \mu_{0} \in X$. However, $T(\tau)$ will in general not map $B_{R}(0)$ into itself. To overcome this difficulty, we introduce the retract $T_{R}: X \rightarrow B_{R}(0)$, 
defined by

$$
\left(T_{R} \mu_{.}\right)_{\circ}=\left(\begin{array}{ll}
\mu_{0} & \text { if } \quad\left\|\mu_{0}\right\| \leqq R \\
\frac{R}{\left\|\mu_{0}\right\|} \mu_{.} & \text {if }\left\|\mu_{0}\right\|>R .
\end{array}\right.
$$

$T_{R} \circ T(\tau)$ certainly maps $B_{R}(0)$ into itself, and we have

Lemma 4.5. $T_{R} \circ T(\tau)$ is continuous on $B_{R}(0)$. For every $\tau \geqq 4 \pi C(\delta), T_{R} \circ T(\tau) B_{R}(0)$ is a uniformly equicontinuous subset of $B_{R}(0)$, and hence relatively compact.

Proof. The crude truncation from Sect. 3.2 is such that the measures $Q^{\delta}\left(\mu_{x}, \mu_{x}\right)$ and $R^{\delta}\left(\mu_{x}\right) \mu_{x}$ are compactly supported in $v^{2}+w^{2} \leqq \delta^{-2}$. From this and equations (4.4-6) one easily sees that the family of functions $\int d v_{x}(v)$, where $v$. is any solution of (4.4-6) with $\mu_{.} \in B_{\rho}(0)$, is uniformly bounded and uniformly equicontinuous. It follows that $T_{R} \circ T(\tau)$ is continuous. The uniform equicontinuity of $T(\tau) B_{R}(0)$ is obvious, because by (4.4-6), for any $\varphi \in C_{\infty}$, we have uniform bounds on the derivatives $\frac{d}{d x}\left\langle v_{x}, \varphi\right\rangle$.

In view of the boundedness and equicontinuity of the set $\left\{\int d\left(T(\tau) \mu_{0}\right)_{x} ; \mu_{0} \in B_{R}(0)\right\}$, the retract $T_{R}$ preserves uniform equicontinuity. This implies the second assertion. q.e.d.

Corollary. $T_{R} \circ T(\tau)$ has a fixed point in $B_{R}(0)$.

Proof. This is an immediate consequence of Lemma 4.2 and Lemma 4.5. q.e.d.

Let $\mu_{0}^{\tau}$ be this fixed point. If $\left\|T(\tau) \mu_{0}^{\tau}\right\| \leqq R, \mu^{\tau}$. is actually a fixed point of $T(\tau)$, because $T_{R}$ is the identity on $B_{R}(0)$. From $(4.4-6)$ one readily verifies that $\mu_{0}^{\tau}$ is then a solution of (3.3-4). Following an idea by Schaefer [8], we show that if $R$ is large enough and $\tau \geqq 4 \pi C(\delta)$, then actually $\left\|T(\tau) \mu^{\tau}\right\| \leqq R$. The key step towards this end is to prove that the set of all fixed points of $T_{R}{ }^{\circ} T(\tau)$ is uniformly bounded.

Let $T_{R} \circ T(\tau) \mu_{\bullet}^{\tau}=\mu_{\bullet}^{\tau}$. Without restricting the generality, suppose that $\left\|T(\tau) \mu_{\bullet}^{\tau}\right\| \geqq R$, i.e.

$$
T(\tau) \mu_{\bullet}^{\tau}=\frac{1}{\lambda} \mu_{\bullet}^{\tau}
$$

where

$$
0<\lambda=\frac{R}{\left\|T(\tau) \mu_{\bullet}^{\tau}\right\|} \leqq 1 .
$$

To simplify notation, we suppress now the dependence of $\mu$ on $\tau$. Explicitly, (4.7) reads

$$
\begin{array}{r}
\left.\mu_{0}\right|_{\{\xi>0\}}=\lambda \mu_{0}^{+},\left.\quad \mu_{a}\right|_{\{\xi<0\}}=\lambda \mu_{a}^{-} \\
\xi \frac{d}{d x} \mu_{x}=0 \text { for }|\xi| \leqq \delta, \quad|\xi| \geqq 1 / \delta,
\end{array}
$$




$$
\xi \frac{d}{d x} \mu_{x}=\tau \rho\left[\mu_{.}\right](x)(\lambda-1) \mu_{x}+\lambda\left(Q^{\delta}\left(\mu_{x}, \mu_{x}\right)-\mu_{x} R^{\delta}\left(\mu_{x}\right)\right)
$$

for $\delta<|\xi|<1 / \delta$.

By the usual conservation laws,

$$
\int \varphi(v) d\left[Q^{\delta}\left(\mu_{x}, \mu_{x}\right)-R^{\delta}\left(\mu_{x}\right) \mu_{x}\right](v)=0,
$$

where $\varphi(v)=1, v$ or $v^{2}$ respectively. Therefore, from (4.8-10), we find

$$
\frac{d j}{d x}=(\lambda-1) \tau \rho^{2},
$$

and, by multiplying (4.10) by $\xi$ and integrating,

$$
\frac{d p}{d x}=(\lambda-1) \tau \rho j
$$

In the sequel, we use the notation $j^{+}=\int_{\xi>0} \xi d \mu_{x}(v), j^{-}=\int_{\xi<0}|\xi| d \mu_{x}(v)$. From (4.11),

$$
j(x)=j^{+}(x)-j^{-}(x) \leqq j^{+}(0)-j^{-}(0) .
$$

For $x=a$, we get,

$$
j^{+}(a)+j^{-}(0) \leqq j^{+}(0)+j^{-}(a)
$$

Let $e(x)=\int v^{2} d \mu_{x}(v)$

$$
q^{+}(x)=\int_{\xi>0} \xi v^{2} d \mu_{x}(v), \quad q^{-}(x)=\int_{\xi<0}|\xi| v^{2} d \mu_{x}(v)
$$

and $q(x)=q^{+}(x)-q^{-}(x)$. From $(4.9-10), \frac{d}{d x} q=(\lambda-1) \tau \rho e \leqq 0$, so also

$$
q^{+}(a)+q^{-}(0) \leqq q^{+}(0)+q^{-}(a) .
$$

Note that the right-hand sides of $(4.14-15)$ are, by (4.8), a priori bounded. By (4.11), $j(x)$ is nonincreasing. To estimate $p(x)$, we distinguish 3 cases:

a) if $j(x) \geqq 0$ for all $x, p(x)$ is also nonincreasing, so $p(x) \leqq p(0)=p^{+}(0)+p^{-}(0)$. $p^{+}(0)$ is given, so in this case we need to estimate $p^{-}(0)$.

b) if $j(x) \leqq 0$ for all $x, p(x)$ is nondecreasing so $p(x) \leqq p(a)=p^{+}(a)+p^{-}(a) \cdot p^{-}(a)$ is given, so here we need an estimate on $p^{+}(a)$.

c) if $j(x)$ changes sign, it can do so at most once. Suppose that $j(x)>0$ for $x \in\left[0, x_{1}\right), j(x)=0$ for $x \in\left[x_{1}, x_{2}\right]$ and $j(x)<0$ for $x \in\left(x_{2}, a\right]$. We see from (4.12) that $p$ is nonincreasing on $\left[0, x_{1}\right)$, constant in $\left[x_{1}, x_{2}\right]$ and nondecreasing on $\left(x_{2}, a\right]$. Also, $p$ is nonnegative by its definition, so

$$
0 \leqq p(x) \leqq \max \{p(0), p(a)\} \text {. }
$$

We realize that a priori bounds on $p$ will follow from a priori bounds on $p^{-}(0)$ and $p^{+}(a)$. By the Cauchy-Schwarz inequality,

$$
p^{-}(0)=\int_{\xi<0} \xi^{2} d \mu_{0}(v)
$$




$$
\begin{aligned}
& \leqq\left(\int_{\xi<0}|\xi| d \mu_{0}(v)\right)^{1 / 2}\left(\int_{\xi<0}|\xi|^{3} d \mu_{0}(v)\right)^{1 / 2} \\
& \leqq\left(j^{+}(0)+j^{-}(a)\right)^{1 / 2}\left(q^{+}(0)+q^{-}(a)\right)^{1 / 2} \\
& =\lambda\left(\int_{\xi<0}|\xi| d \mu_{a}^{-}+\int_{\xi>0} \xi d \mu_{0}^{+}\right)^{1 / 2}\left(\int_{\xi<0}|\xi| v^{2} d \mu_{a}^{-}+\int_{\xi>0} \xi v^{2} d \mu_{0}^{+}\right)^{1 / 2} .
\end{aligned}
$$

The last two estimates follow from (4.14), (4.15) and (4.8). The same estimate applies to $p^{+}(a)$. Let

$$
C\left(\mu_{0}^{+}, \mu_{a}^{-}\right)=\left(\int_{\xi<0}|\xi| d \mu_{a}^{-}+\int_{\xi>0} \xi d \mu_{0}^{+}\right)^{1 / 2}\left(\int_{\xi<0}|\xi| v^{2} d \mu_{a}^{-}+\int_{\xi>0} \xi v^{2} d \mu_{0}^{+}\right)^{1 / 2} .
$$

We have proved

Lemma 4.6. For any solution $\mu^{\tau}$ of the equation

$$
T_{R} \circ T(\tau) \mu_{\bullet}^{\tau}=\mu_{\bullet}^{\tau}
$$

we have

$$
\max _{x \in[0, a]} \int \xi^{2} d \mu_{x}^{\tau}(v) \leqq C\left(\mu_{0}^{+}, \mu_{a}^{-}\right)+p^{+}(0)+p^{-}(a) .
$$

Remarks. 1. We emphasize that $C\left(\mu_{0}^{+}, \mu_{a}^{-}\right)$is independent of the truncation parameter $\delta$. Estimates of $p(x)$ which are $\delta$-dependent can be obtained as in [1] and take the form

$$
p(x) \leqq p^{+}(0)+p^{-}(a)+K(\delta)\left(j^{+}(x)+j^{-}(x)\right)
$$

(use that for $|\xi|<\delta$ and $|\xi|>1 / \delta \mu_{x}$ is just given by the data). By using the monotonicity argument from above and the a priori bound (4.14), one obtains a $\delta$-dependent a priori estimate on $p(x)$. Equation (4.15) is not needed for this estimate, but the result from Lemma 4.6 is more promising because it remains useful as $\delta \rightarrow 0$.

2. The independence of the bounds on $p(x)$ from $\delta$ is what enables us to prove a result for the truncation described in Sect. 3.1. We return to this in Sect. 5.

3. If $\left\|T(\tau) \mu^{\tau}\right\|<R$, the discussion starting with (4.7) goes through with $\lambda=1$.

Theorem 4.7. For any $\delta>0$, the problem (3.3-4) has a measure solution, i.e. a $\mu_{\bullet} \in X$ such that

$$
\xi \frac{d}{d x} \mu_{x}=C^{\delta}\left(\mu_{x}, \mu_{x}\right)
$$

and

$$
\left.\mu_{0}\right|_{\{\xi>0\}}=\mu_{0}^{+},\left.\quad \mu_{a}\right|_{\{\xi<0\}}=\mu_{a}^{-} .
$$

Proof. It is clearly enough to find a fixed point of $T(\tau)$ for some $\tau$. By (4.18), for $\mu_{\text {. }}^{\tau}$ as in Lemma 4.6,

$$
\begin{aligned}
\left\|\mu_{0}^{\tau}\right\| & \left.\leqq \sup _{\substack { x \in[0, a] \\
\begin{subarray}{c}{0<\xi<\delta \\
1 / \delta<\xi{ x \in [ 0 , a ] \\
\begin{subarray} { c } { 0 < \xi < \delta \\
1 / \delta < \xi } }\end{subarray}} d \mu_{0}^{+}+\int_{\substack{-\delta<\xi<0 \\
-1 / \delta>\xi}} d \mu_{a}^{-}+\frac{1}{\delta^{2}} \int \xi^{2} d \mu_{x}^{\tau}\right) \\
& \leqq C_{1}(\delta) .
\end{aligned}
$$

Here, $C_{1}(\delta)$ is a constant which depends on the boundary values and on $\delta$, but 
not on $\tau$ or $R$. Therefore, all fixed points of $T_{R} \circ T(\tau)$, where $\tau \geqq 4 \pi C(\delta)$, are inside some bounded set $B_{R_{1}}(0)$ for all $R$. Let $\tau_{1}:=4 \pi C(\delta)$, then $T\left(\tau_{1}\right) B_{R_{1}}(0)$ is contained in some $B_{R_{2}}(0)$, such that

$$
T_{R_{2}} \circ T\left(\tau_{1}\right) \mu_{\bullet}^{\tau_{1}}=\mu_{\bullet}^{\tau_{1}} .
$$

As $\left\|\mu_{0}^{\tau_{1}}\right\| \leqq R_{1},\left\|T\left(\tau_{1}\right) \mu_{0}^{\tau_{1}}\right\| \leqq R_{2}$, i.e. $T\left(\tau_{1}\right) \mu_{0}^{\tau_{1}}=\mu_{0}^{\tau_{1}}$. This completes the proof. q.e.d.

\section{The Main Theorem}

We now return to the minimally truncated problem from Sect. 3.1. Consider, for any $\delta>0$, the solutions of

$$
\begin{aligned}
\xi \frac{d}{d x} \mu_{x} & =C^{\delta}\left(\mu_{x}, \mu_{x}\right), \\
\left.\mu_{0}\right|_{\{\zeta>0\}} & =\mu_{0}^{+},\left.\quad \mu_{a}\right|_{\{\xi<0\}}=\mu_{a}^{-},
\end{aligned}
$$

which we just discussed. As in (2.2), we write (5.1) in measure notation as

$$
\begin{aligned}
& -\int_{0}^{a} \int_{0} \frac{\partial}{\partial x} \varphi(x, v) \xi d \mu_{x}^{\delta}(v) d x-\int_{\xi>0} \varphi(0, v) \xi d \mu_{0}^{+}(v)+\int_{\xi<0} \varphi(a, v) \xi d \mu_{a}^{-}(v) \\
& \quad=\int_{0}^{a} \int_{v} \int_{w} \int_{n}\left(\varphi^{\prime}-\varphi\right) B_{\varepsilon}^{\delta}(v, n, w) d n d \mu_{x}^{\delta}(v) d \mu_{x}^{\delta}(w) d x .
\end{aligned}
$$

We have now written $\mu_{x}^{\delta}$ rather than $\mu_{x}$ to emphasize the dependence on $\delta$.

By (4.18), $\sup _{x \in[0, a]} \int \xi^{2} d \mu_{x}^{\delta}(v) \leqq C\left(\mu_{0}^{+}, \mu_{a}^{-}\right)+p^{+}(0)+p^{-}(a)$. From these bounds, it is clear that the measures $\left\{\xi^{2} \mu_{x}^{\delta}\right\}_{\delta>0}$ form a weak-* relatively compact set. By using the usual Cantor diagonalization, we can find a sequence $\delta_{n} \rightarrow 0$ such that $\xi^{2} \mu_{x}^{\delta_{n}} \rightarrow \tilde{\mu}_{x}$ for all $x \in A$, where $A$ is a countable dense subset of $[0, a]$, and $\tilde{\mu}_{x}$ is a measure on $\mathbb{R}^{3}$ such that $\int d \tilde{\mu}_{x}(v) \leqq C\left(\mu_{0}^{+}, \mu_{a}^{-}\right)+p^{+}(0)+p^{-}(a)$. By using the definition of admissible test function and the conditions imposed in Sect. 3.1, we can rewrite $(5.3)$ as

$$
\begin{aligned}
& -\int_{0}^{a} \int\left(\frac{\partial}{\partial x} \varphi(x, v) / \xi\right) \xi^{2} d \mu_{x}^{\delta}(v)+\int_{\xi<0} \varphi(a, v) \xi d \mu_{a}^{-}(v)-\int_{\xi>0} \varphi(0, v) \xi d \mu_{0}^{+}(v) \\
& =\int_{0}^{a} \iint_{v} \int_{w}\left(\varphi^{\prime}-\varphi\right) B(n, v-w) k_{\delta} \cdot \chi_{\varepsilon} /\left(\xi^{2} \xi_{*}^{2}\right) d n \xi^{2} d \mu_{x}^{\delta}(v) \xi_{*}^{2} d \mu_{x}^{\delta}(w) d x .
\end{aligned}
$$

Equation (5.4) implies that $\left\{\xi^{2} \mu_{x}^{\delta}\right\}_{\delta}$ is an equicontinuous family of measure-valued functions, so $\xi^{2} \mu_{x}^{\delta_{n}} \rightarrow \tilde{\mu}_{x}$ for actually all $x \in[0, a]$, where $\tilde{\mu}_{0} \in X$. By taking the limit $\delta_{n} \rightarrow 0$ in (5.4), we have

$$
\begin{gathered}
-\int_{0}^{a} \int_{0} \frac{\partial}{\partial x} \varphi(x, v) / \xi d \tilde{\mu}_{x}(v)-\int_{\xi>0} \varphi(0, v) \xi d \mu_{0}^{+}(v)+\int_{\xi<0} \varphi(a, v) \xi d \mu_{a}^{-}(v) \\
=\int_{0}^{a} \iint_{v} \int_{w n}\left(\varphi^{\prime}-\varphi\right) B(n, v-w) \cdot \chi_{\varepsilon} /\left(\xi^{2} \xi_{*}^{2}\right) d n d \tilde{\mu}_{x}(v) d \tilde{\mu}_{x}(w) d x .
\end{gathered}
$$

The measure function $\mu_{0}=\left(1 / \xi^{2}\right) \tilde{\mu}$. is then possibly unbounded, but it satisfies (2.2) with $B$ replaced by $B_{\varepsilon}$. We have thus proved our main result: 
Theorem 5.1. There is a measure-valued function

$$
x \rightarrow \mu_{x}
$$

such that $x \rightarrow \xi^{2} \mu_{x}=\tilde{\mu}_{x}$ is continuous, takes values in $M$ and satisfies (5.5). In this

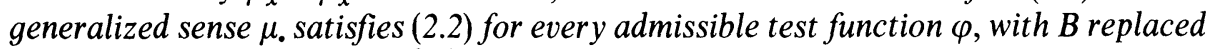
by $B_{\varepsilon}$. In particular if $B_{\varepsilon} / \xi^{2} \xi_{*}^{2}=0$ for $\xi=0$, then $\mu$. satisfies (2.2) in the original sense with $B=B_{\varepsilon}$.

Remark. Obviously, the method used in Sects. 4 and 5 applies to many other interesting boundary conditions (see [1], where such conditions were dealt with for the discrete velocity case). As an example, we mention the problem where $\left.\mu_{0}\right|_{\{\xi>0\}}$ is still given as before such that $\int_{\xi>0} d \mu_{0}$ and $\int_{\xi>0} \xi v^{2} d \mu_{0}$ are finite, but at $a$, we replace the boundary condition by specular or reverse reflection (i.e. $\left.\mu_{a}\right|_{A}=\left.\mu_{a}\right|_{A^{\prime}}$, where $A \subset\{\xi>0\}$ is measurable and $A^{\prime}$ is the image of $A$ under specular or reverse reflection - in particular, $\left.A^{\prime} \subset\{\xi<0\}\right)$. Then for all fixed points of the operators $T_{R} \circ T(\tau)$,

and

$$
\begin{aligned}
\int \xi d \mu_{a} & =\int \xi v^{2} d \mu_{a}=0 \\
\int_{\xi<0}|\xi| d \mu_{0} & \leqq \int_{\xi>0} \xi d \mu_{0},
\end{aligned}
$$

$$
\int_{\xi<0}|\xi| v^{2} d \mu_{0} \leqq \int_{\xi>0} \xi v^{2} d \mu_{0} .
$$

All the assertions from Sects. 4 and 5 hold if we restrict the class of test functions $\varphi$ such that $\varphi$ has the same symmetry at $a$ as $\mu_{a}$.

Acknowledgement. This research was supported by the Natural Sciences and Engineering Research Council of Canada under grand nr. A 7847

\section{References}

1. Cercignani, C., Illner, R., Shinbrot, M.: A boundary value problem for discrete-velocity models. Duke Math. J. 55 (4), 889-900 (1987)

2. Cercignani, C., Illner, R., Shinbrot, M.: A boundary value problem for the two-dimensional Broadwell model. Commun. Math. Phys. 114, 687-698 (1988)

3. Cercignani, C., Illner, R., Shinbrot, M., Pulvirenti, M.: On nonlinear stationary half-space problems in discrete kinetic theory. J. Stat. Phys. 52 (3/4), 885-896 (1988)

4. Coron, F., Golse, F., Sulem, C.: Classification of kinetic layer problems. Commun. Pure Appl. Math. 41, 409-435 (1988)

5. Illner, R.: On steady boundary value problems in discrete kinetic theory, and an application to digital image processing. In: Discrete Kinetic Theory, Lattice Gas Dynamics and Foundations of Hydrodynamics. Singapore: World Scientific 1989

6. Reed, M. C., Simon, B.: Methods of Modern Mathematical Physics I. New York: Academic Press 1980

7. Royden, H. L.: Real Analysis. New York: Macmillan 1968

8. Schaefer, H.: Ueber die Methode der a-priori Schranken. Math. Ann. 129, 415-416 (1955)

9. Smart, D. R.: Fixed Point Theorems. New York: Cambridge University Press 1974

10. Tychonoff, A.: Ein Fixpunktsatz. Math. Ann. 111, 767-776 (1935) 